\title{
Titan: Earth-like on the Outside, Ocean World on the Inside
}

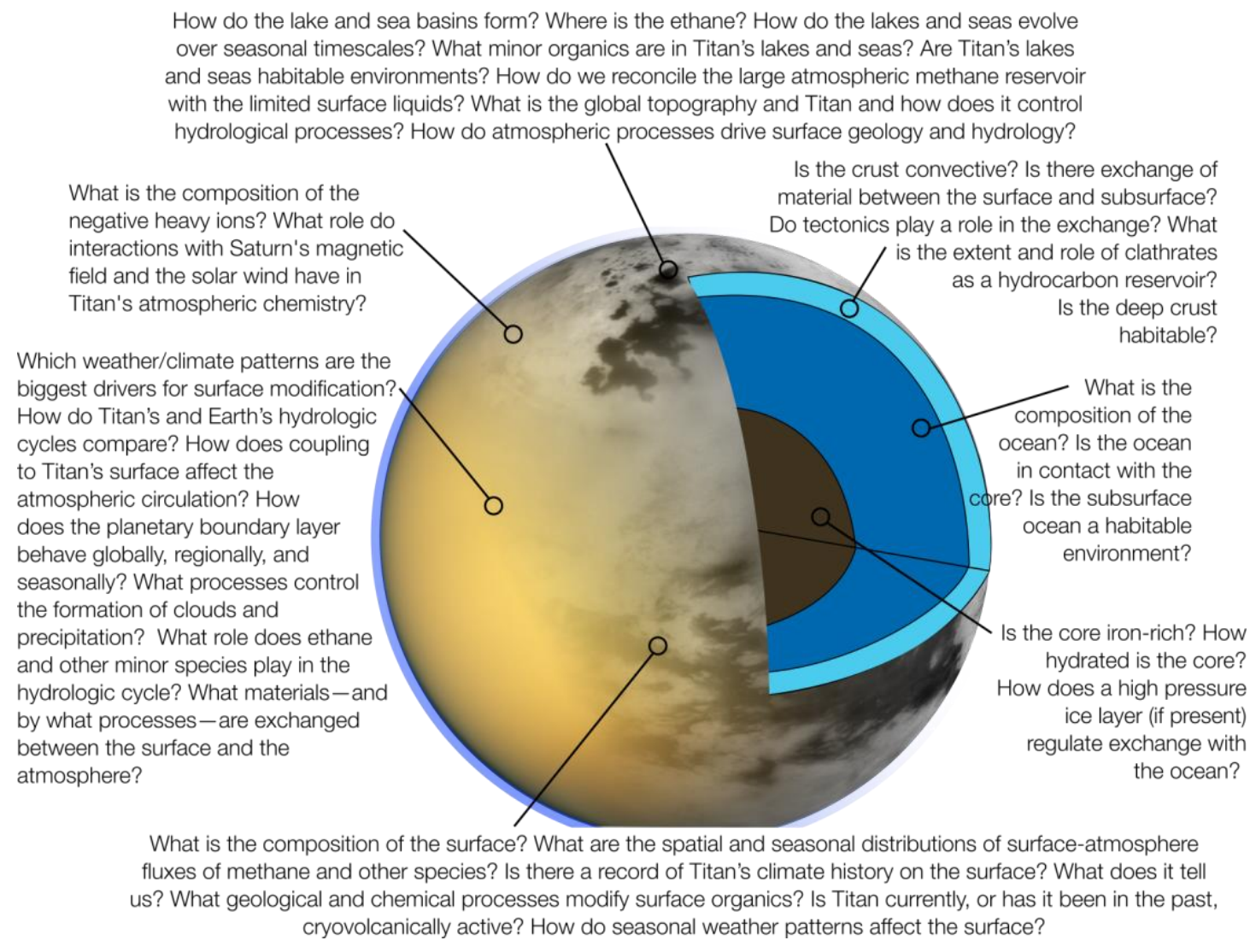

Shannon M. MacKenzie*1, Sam Birch2, Sarah Hörst3, Christophe Sotin4, Erika Barth5, Juan Lora6, Melissa G. Trainer7, Paul Corlies2, Michael J. Malaska4, Ella Sciamma-O'Brien8, Alexander E. Thelen7, Elizabeth Turtle1, Jani Radebaugh, Jennifer Hanley 10, Anezina Solomonidou11, Claire Newmann12, Leonardo Regoli1, Sebastien Rodriguez13, Benoit Seignovert4, Alex Hayes14, Baptiste Journaux15, Jordan Steckloff16, Delphine Nna-Mvondo17, Thomas Cornet18, Maureen Palmer19, Rosaly Lopes4, Sandrine Vinatier20, Ralph Lorenz1, Conor Nixon7, Ellen Czaplinski21, Jason W. Barnes22, Ed Sittler7, Andrew Coates23

1Johns Hopkins University Applied Physics Lab; 2Massachusetts Institute of Technology; 3Johns Hopkins University; 4Jet Propulsion Laboratory; 5 Southwest Research Institute, Boulder; 6 Yale University; 7NASA Goddard Space Flight Center; 8NASA Ames; 9 Brigham Young

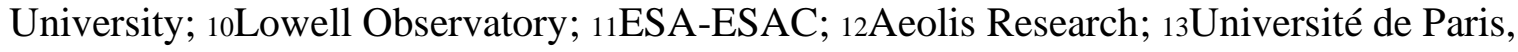
Institut de Physique du Globe de Paris, CNRS; 14 Cornell University; 15 University of Washington; 16Planetary Science Institute; 17University of Maryland, NASA GSFC; 18Aurora Technology B.V. for ESA; 19 University of Arizona; 20LESIA, Observatoire de Paris, Université PSL, CNRS, Sorbonne Université, Université de Paris; 21 University of Arkansas; 22 University of Idaho, 23Mullard Space Science Laboratory, University College London

*shannon.mackenzie@jhuapl.edu (240) 592-1521 
Thanks to the Cassini mission, the pale orange dot of Pioneer and Voyager encounters has been revealed to be a dynamic, hydrologically-shaped, organic-rich ocean world offering unparalleled opportunities to explore prebiotic chemistry. And while Cassini-Huygens revolutionized our understanding of each of the three "layers" of Titan - the atmosphere, the surface, and the interior - we are only beginning to hypothesize how these realms interact. In this white paper, we

- summarize the current state of Titan knowledge,

- discuss how future exploration of Titan would address some of the next decade's most compelling planetary science questions, and

- demonstrate why Titan exploration, both with and beyond the Dragonfly New Frontier's mission, is a necessary and complementary component of an Ocean Worlds Program that seeks to understand whether habitable environments exist elsewhere in our solar system.

\section{TITAN IS AN ORGANIC WORLD}

Titan hosts the most Earth-like atmosphere in the solar system. Similarities include the atmospheric structure[1], a nitrogen-dominated composition $\left(95 \% \mathrm{~N}, 4 \% \mathrm{CH}_{4}\right.$, and $1 \%$ trace species at the surface), and a surface pressure of 1.5 bar. The photolytic destruction of atmospheric methane initiates a chain of photochemical reactions[2] responsible for the plethora of organic species that make up Titan's haze observed by Cassini-Huygens and ground-based facilities[3-17]]. While unlike present-day Earth, this may be similar to the haze production on Early Earth.

Pressing Questions and Future Investigations: Cassini identified the compositions of neutral and positive ions up to $99 \mathrm{Da}$ were identified but could only detect the presence of negative ions with a mass-to-charge ratio so large as to be similar to that of terrestrial proteins[18-21]. These molecules grow larger during polar winter and with decreasing altitude[22], but their ultimate fate remains unknown. Thus, our current list of known compounds represents only the tip of the organic factory iceberg. Ongoing modeling efforts and laboratory experiments continue to investigate what reactions might be at work[23,19,24-48] and to explore the properties of Titan haze analogs, tholins [49,51-54]. However, determining the dominant chemical pathways of Titan's organic factory will require a more complete understanding of the chemical composition, abundance, and distribution of organic hazes in the atmosphere, especially at different seasons in Titan's year (29.5 Earth years).

Beyond chemical pathways, many questions remain or have arisen from Cassini. How seasonal trends in condensate distributions extend to lower altitudes[11,55] and whether these affect sedimentation onto the surface remains unknown. Models indicate that microphysics plays an important role in cloud and haze formation; increased understanding of the distribution, optical properties, and composition of hazes and clouds from observational (at Titan and ground-based) and lab data would constrain physical models.

Several approaches to constrain the age of Titan's atmosphere overlap at $\sim 300-500 \mathrm{Myr}$, but whether this indicates the age of the atmosphere or ongoing methane photolysis is unclear[2]. Replenishment from the interior offers a compelling solution to the methane loss, but more data (crustal dynamics, surface feature identification, isotopic fractionation, high-degree gravity field) are required to evaluate the likelihood of candidate mechanisms like clathrate dissociation and cryovolcanism[56-59]. 
Role in an Ocean Worlds Program: Atmospheric organic species are the ultimate source of the organic sediments that dominate Titan's surface, so their formation and evolution in the atmosphere have important implications for surface geology and possible subsurface nutrient availability. Furthermore, investigating the processes that create complex species in Titan's atmosphere - without, presumably, biological catalysts like those responsible for large molecules here on Earth - offers fundamental insight into the chemistry that may precede or facilitate the rise of biochemistry on Early Earth[60,61] and beyond. The study of Titan's atmospheric chemistry therefore offers crucial context for the habitability potential of other ocean worlds where the essential elements may be less abundant.

Relevance to other planets: Questions surrounding the dynamics and longevity of Titan's atmosphere link to questions about the gas and ice giants[62,63] and - given the coupling between the atmosphere and surface - about Earth, Venus, Mars, and Pluto[64-72]. Without its own magnetic field, Titan's interactions with the solar wind and Saturn's magnetosphere offer the opportunity to explore whether magnetic fields are necessary for habitability. Moreover, Titan's atmosphere serves as a powerful backyard analog for hazy exoplanets - from understanding the formation and evolution of atmospheric aerosols to how we might best detect and observe them - as we have ground truth from both remote and in situ sensing[73-85].

\section{TITAN IS AN ACTIVE HYDROLOGICAL AND SEDIMENTARY WORLD}

Titan also has a methane-based hydrologic cycle akin to Earth's[86,87]. The seasonal timing and magnitude of surface-atmosphere fluxes of methane, including observed clouds and rainstorms [88-92], are probably linked to existing surface and subsurface liquid reservoirs [93,86,94-100,71]. The hydrological cycle shapes the surface, producing landforms that bear a striking resemblance to those found on Earth. Lakes and seas of liquid methane and ethane[101-105] up to hundreds of meters deep[102-104] are found across Titan's polar regions[88,106-111] and are currently evolving[89,91,92,109,112-116]. River channels and rounded cobbles imaged by Huygens [117] and the radar-bright channels[118-125] and fans [126-128] observed by Cassini[129,130] evidence that Titan's hydrological cycle is intimately connected with the sedimentary cycle: complex organic compounds synthesized and advected in and by the atmosphere are further transported and modified across the surface by the only known active extraterrestrial hydrologic cycle.

Perhaps the best studied sediments on Titan are the organic sands that occupy $17 \%$ of the moon's surface [131-137]. Linear dunes (100s of kilometers long and $\sim 100 \mathrm{~m}$ in height) demonstrate the importance of aeolian processes and underlying topography in the redistribution of Titan's organics[136,138-142]. Titan's vast mid-latitude plains ( $\sim 65 \%$ of the surface) are also hypothesized to consist of organic materials[143-146], but their composition and origin remains unknown.

Pressing Questions and Future Investigations: Despite advances in our understanding of the landscapes of Titan, the composition of the surface remains ill-constrained. Cassini observations, limited in spectral and spatial resolution, suggest two general categories of materials: organicrich and water-ice rich[131,136,137,146-148]. Continued laboratory and theoretical work into the possible compositions and physical properties of Titan surface materials are crucial for informing interpretations of Cassini data[149-152] and supporting future exploration of the surface like the Dragonfly mission.

Dramatic advances in our understanding of Titan's seasonally-evolving weather and climate $[93,86,87,71]$ are similarly accompanied by new questions and key unknowns: the principal 
mechanisms controlling the timing and distribution of humidity, convection, methane cloud formation, and precipitation remain incompletely understood, as do the sources and sinks of atmospheric methane and the roles of atmospheric variability and transient phenomena like dust storms [153] in the climate. Likewise, the impact of heterogeneous surface-atmosphere couplingfor example, how Titan's lakes affect the north polar environment — and the magnitude and importance of regional climate variability are still largely unexplored. Further observations of Titan's weather phenomena, coupled with improvements in physical modeling, are needed to continue elucidating Titan's climate system and to link synoptic-scale processes to those at global and interannual scales, as well as to their impacts on the surface.

Global, high-resolution imaging $(<100 \mathrm{~m})$ would revolutionize our understanding of Titan's landforms, how they interact with each other and the atmosphere, and how they have evolved in the same way that Mars Global Surveyor's orbital campaign fundamentally changed the study of Mars. Similarly, the lack of knowledge of Titan's topography specifically limits study of the transport of liquids and sediments on the surface, as well as of the influence of the surface on the atmosphere - Cassini data covers only $9 \%$ of Titan at scales too coarse for detailed hydrological analysis of hydrologic catchments, mountain wave effects, or orographic clouds and precipitation[154]. In conjunction with maps of surface composition at high spatial and spectral resolution, global imaging and topographic data would address fundamental questions surrounding the hydrological, sedimentological, and meteorological cycles of Titan, augmenting Cassini data and complementing Dragonfly's planned local in situ investigations.

Role in an Ocean Worlds Program: Titan represents the organic-rich endmember of the Ocean World spectrum. Understanding the surface and atmospheric processes that create, modify, and transport these materials on Titan, and the timescales and volumes on which they act, would elucidate the role these processes play in planetary habitability and their significance.

Relevance to other planets: Titan's surface and climate system serves as a natural laboratory for studying the fundamentals of a planetary-scale hydrologic cycle and how it controls the physical and chemical evolution of the landscape in an environment akin to but less complex than Earth's. For example, sea level rise is likely ongoing and has dramatically shaped the coasts of Titan's large seas $[155,109,156,89,157,158,116,159]]$ and is likely ongoing although the rates remain loosely constrained; study of Titan's coasts and ongoing erosional/depositional processes could be directly compared to the rapid changes on Earth and inform the study of paleo coastlines on Mars.

\section{TITAN IS AN OCEAN WORLD}

A subsurface water ocean lies beneath Titan's organic-covered ice crust[160], evidence for which includes gravitational tides[161,162] and larger-than-expected obliquity[163,164].

Pressing Questions and Future Investigations: The extent of Titan's crust is loosely constrained to 50-200 km[59,165-167] and the extent and duration of convection within the ice crust $[166-168]$ is still debated. Estimates of the oceanic depth span 500-700 km[161,169-171] and the state of differentiation in the core is unknown[160,164,170,172]. The presence of salts or ammonia may explain the ocean's high density[162,173], but magnesium sulfate is also a potential solution[174]. Primordial icy bodies provided noble gases and organic matter during Titan's accretion, making the interior an even vaster source of organics than the atmosphere, with some models predicting 
1000x the current atmospheric methane abundance[175]. These considerations, coupled with detection of radiogenic 40Ar by Huygens [176] suggest that outgassing from the interior may be responsible for the atmosphere. New isotopic measurements of noble gases and methane are necessary to resolve key questions concerning the ocean composition, the evolution of the interior and atmosphere, and the formation of Titan[177-181].

At pressures $>500 \mathrm{MPa}$, a layer of high-pressure ice may separate Titan's core from the ocean[174], but if the heat flux is high enough and/or salinity high enough, the ocean may be in direct contact with the silicate core[182]. Initially, the presence of high-pressure ice prompted the oceans of the largest icy satellites to be deemed inhospitable, assuming that separation by ice precluded exchange between the ocean and core. However, advances in our knowledge of how ices behave at high pressure show that convection can move material through the ice layer[183,184], including salts and volatiles like $40 \operatorname{Ar}[185,186]$. More laboratory and theoretical investigations [187] into the properties of high pressure ices and hydrates are needed before we fully understand their implications on Ocean World habitability.

Role in an Ocean Worlds Program: Determining whether Titan's ocean is in contact with the rocky core would provide a key constraint to the formation and longevity of large Ocean Worlds both within and beyond our solar system[188]. Studying the very origins of Titan's organic cycle - from the primordial to hydrothermally altered material—informs our understanding of the role of volatile-rich ices in the early solar system.

\section{IS TITAN A HABITABLE WORLD?}

The search for life elsewhere in the universe logically employs the guide of Earth's biochemical foundations of Earth's biosphere, giving rise to the classical conditions necessary for habitability: liquid water, essential elements1, and energy sources[189-191]. All three factors exist on Titan. The question is where they have been or may be collocated and for how long.

Pressing Questions and Future Investigations: Titan's deep crustal ice and subsurface ocean could be one of the largest habitable realms in the solar system, with a volume of liquid water $18 x$ that of the Earth's oceans and CHNOPS 1 potentially available from primordial and/or thermally processed materials[181]. Tectonic activity may facilitate the delivery of surface organics through the crust. Whether any or all of these processes are at work and on what timescales they operate on Titan remain open questions, with implications for other ocean worlds where habitability may rely even more heavily upon the exchange of surface and subsurface material. For example, temperature and pressure conditions at the putative depth of Titan's stagnant lid/convective ice transition are very similar to those encountered within terrestrial deep glacial ice, which hosts a diversity of microbial life[192] in the intergrain channels between solid ice grains[193,194]. In these intergrain regions, microbial metabolism is slow enough that the environment may be habitable for 10,000 years - only a few orders of magnitude lower than Titan's hypothesized convective cycle.

A frigid ambient temperature of $~ 90 \mathrm{~K}_{[195]}$ makes Titan's surface largely inhospitable for Earthlike life, which uses water as the biochemical solvent. However, there are ephemeral scenarios in which liquid water is present at Titan's surface: lavas erupting from cryovolcanoes and impactgenerated melt. While some geomorphological evidence supports the existence of cryovolcanism[145,196-198], its mechanics [199,200] are not well understood, in part due to the lack of

1 E.g. CHNOPS: Carbon, Hydrogen, Nitrogen, Oxygen, Phosphorous, and Sulfur 
constraints on the extent, makeup, and activity of the crust as well as the ocean composition. However, impact craters are found across Titan's surface[201-209]. During the impact, crustal material and surface organics mix; the resulting pockets of liquid water eventually freeze on timescales loosely constrained to up to 10,000s of years[210-215]. Mixing tholins with liquid water in the laboratory produces amino acids on a timescale of days[216-218]. Titan's transient liquid water environments are thus extraterrestrial laboratories for exploring how far prebiotic chemistry can progress under time and energy constraints that are difficult to realistically reproduce experimentally[219]. The Dragonfly mission will take advantage of this opportunity with surface composition measurements near a large impact crater.

Without an understanding of the chemical processes necessary for the emergence of life, it is impossible to say with certainty how long it takes for life to arise[220]. This timescale is a critical unknown in our concept of habitability: is there a minimum time necessary for all the key ingredients to be collocated? The answer to this question has immediate implications for strategizing the search for life elsewhere (both where to search and whether to target extant or extinct life), especially since the lifetime of the liquid oceans on both confirmed and candidate ocean worlds remains an active area of research[221,222]. Any constraints on habitability timescales from Titan's transient liquid water environments would provide key context for exploration of potentially habitable environments and the search for life.

Finally, Titan's lakes and seas of liquid hydrocarbons offer a unique opportunity to investigate whether the solvent necessary for biochemistry must be water. Theoretical considerations suggest alternative chemistries are possible[223,224] and the abundance of solid and liquid organic molecules available on the surface and lack of UV radiation make the surface of Titan an advantageous place for exploring the possibility of a true second genesis [225]. Theoretical investigations are exploring both the possibilities for lipid membrane-like structures in low temperature environments and whether cell membranes are even necessary[13,226-228]. Laboratory and theoretical models are revolutionizing our understanding of the possible conditions within Titan's lakes and seas[9,37,229-238]. Employing these new findings to constrain the habitability potential of Titan's liquid hydrocarbons requires both determining the composition of Titan sediments - as the Dragonfly mission's plans to do by exploring at a portion of one of Titan's low-latitude dune fields - and monitoring the composition, physical conditions, and seasonal evolution of Titan's polar lakes and seas with future missions.

\section{FUTURE INVESTIGATIONS AT TITAN}

Dragonfly, the next New Frontiers (NF) mission, is a relocatable lander, to explore the prebiotic chemistry of Titan's surface[239,240]. Arriving in 2034, Dragonfly will answer a critical unknown: the chemical composition of Titan's solid sediments. By using a mass spectrometer to measure compositions of the organic-rich sands of the equatorial dune fields, water-ice rich clasts from the relatively unaltered interdunes, and previously melted impact melt ejecta from an impact crater, Dragonfly will begin to answer the question of how far prebiotic chemistry can progress in environments that provide long-term access to key ingredients for life, thereby providing critical context for astrobiological investigations across the solar system. Dragonfly will also determine elemental abundances will be determined in the near subsurface beneath the lander with a gamma ray neutron spectrometer, thus informing the availability and distribution of elements key to habitability. 
Sample provenance both at the scale of Dragonfly's immediate environs and the local region is key to interpreting the chemical findings in context. Dragonfly is thus equipped with a suite of cameras to conduct imaging campaigns at local, nested scales. Meteorological and geophysical instruments will determine aeolian transport rates and monitor local weather conditions, as well as probing the thermal and electrical properties of the surface. Geophones and a seismometer round out the contextual measurements by probing the dynamics and properties of the ice crust, potentially constraining the depth to the ocean[241].

Dragonfly's payload is thus poised to revolutionize not only our understanding of Titan's chemistry and geology but address more broadly how far prebiotic chemistry can progress and what chemical and geological processes make a planet or moon habitable. But, just as Curiosity addresses different fundamental science than $M R O$, the NF-scope and architectural choices that make Dragonfly best suited for its local in situ investigation necessarily preclude addressing many other outstanding questions at Titan, especially those requiring a global perspective.

Thus, as demonstrated by exploration of Mars, a sequence of opportunities is needed to build upon and sufficiently leverage the detailed exploration of Titan begun by Cassini-Huygens and to be continued by Dragonfly in the coming decades. In particular, exploring the polar lakes and seas, their influence on Titan's global hydrologic cycle, and the potential habitability, will remain out of even Dragonfly's impressive range. Such measurements would also be complemented by orbital imaging at higher spatial and temporal resolutions than what Cassini or ground-based observations could provide. A higher order gravity field would reveal eroded craters and thus constrain the prevalence of transient liquid water environments. More specifically, Dragonfly's seismic investigation of the interior would be significantly enhanced by a global topographic dataset and higher fidelity mapping of the gravity field. Further study of the dynamics of Titan's climate and the seasonal evolution of hazes and weather phenomena (e.g. clouds and haboobs239,241-246) requires continued long-term monitoring with ground- and space-based assets as Titan's northern summer unfolds. A global imaging dataset would facilitate understanding the beginning-to-end life cycle of the materials sampled by Dragonfly. For additional investigations and options for how they might be manifested in missions to be planned in the next decade, please see white papers by Barnes et al. and Nixon et al.

\section{TITAN IS AN UNPARALLELED DESTINATION}

Titan offers the opportunity to study a myriad of fundamental planetary science questions. The processes that govern its atmosphere, surface, and interior and interactions between these three environments make Titan an analog for destinations across the solar system and beyond. In the next decade, Dragonfly will continue the legacy of Cassini-Huygens and radically transform our understanding of Titan's chemistry, geology, and astrobiological potential. But if the last decade has taught us anything, it's that this moon's complexity tends to defy our imagination. There is still much left to learn before we fully understand Saturn's largest moon, requiring mission opportunities in addition to Dragonfly in the next decade.

HyerlinKed ReferenCES 1 Fulchignoni, Nature (2005). 2 Hörst, JGRP (2017). 3Marten, Icarus (2002). 4Gurwell, ApJ (2004). 5Ali, P\&SS (2013). 6Cordiner AJL (2014).7Cordiner, AJL (2015).8Cordiner, AJL (2018). 9 Cordier, Nat Geo (2019).10Molter AJ (2016). 11Desai ApJL (2017).12Lai AJ (2017). 13Palmer Sci Adv (2017).14Teanby AJ 
(2007).20Wellbrock GRL (2013).21Woodson JGRSP (2015).22Wellbrock MNRAS (2019). 23Rannou Icarus (2004). 24Vuitton Icarus (2007).25Vuitton (2019). 26Krasnopolsky Icarus (2009). 27Krasnopolsky (2014). 28Nixon AJ (2012). ${ }_{29}$ Waite P\&SS (2013). 30Larson Icarus (2014). 31 Dobrijevic (2014). 32 Dobrijevic (2016). 33Hickson A\&A (2014). 34Lara A\&A (2014). 35 Loison Icarus (2015). 36Loison Icarus (2019). 37Luspay-Kuti ApJ (2015). 38Wong Icarus (2015). 39Sebree Icarus (2016). 40Barth P\&SS (2017). 41Douglas Icarus (2018). 42,43 Mukundan ApJ (2018). 44Berry ACS ESC (2019). 45Bourgalais JGRSP (2019). 46Dubois ApJ (2019). 47Dubois Icarus (2019). 48 Dubois Icarus (2020). 49Cable Chem Rev (2012). 50Rannou A\&A (2019). 51Gautier P\&SS (2017). 52Sciamma-O’Brien Icarus (2017). 53Hörst Icarus (2018). 54Sebree ApJ (2018). 55Coates P\&SS (2009).56Lunine Icarus (1987).57Tobie Nature (2006). 58Choukroun Icarus (2010). 59 Choukroun GRL (2012). 60 Trainer PNAS (2006). 61 Trainer Cur. Org. Chem. (2013). 62Robinson Nat Geo (2014). 63Toledo Icarus (2019). 64Mitchell AJ (2014). 65Mandt Icarus (2015).66Brain JGRP (2016). 67Guendelman GRL (2018). 68Read An. Rev. of Earth \& Plan. Sci.(2018). 69 Crismani JGRSP (2019). 70Köhn Icarus (2019). 71Faulk Nat. Astro. (2020). 72Kite P\&SS (2020).73de Kok E\&PA (2012). 74Forget Phil. Trans. RSL A (2014). 75Tokano Ori. of Life and Evol. of the Bios. (2015). 76Arney Astrobio (2016). 77 Checlair P\&SS (2016).78García Muñoz Nat. Astro. (2017). 79He AJL (2017). 80Hörst Nat. Astro. (2018). 81Levi AJ (2019). 82Lora AJ (2018).83Alvarez Navarro Euro Phys J Plus (2019).84Martínez-Rodríguez AJ (2019).85Miguel MNRAS (2019).86Mitchell Annu. Rev. Earth Planet. Sci (2016).87Hayes Nat. Geo. (2018). 88 Turtle GRL (2009). 89Turtle Science (2011). 90 Turtle GRL (2011). 91Barnes AJ (2013). 92Dhingra GRL (2019). 93Mitchell JGRP (2008). 94Tokano Astrobio. (2009). 95Tokano JGRP (2019). 96Tokano GRL (2020).97Lora GRL (2015). 98Lora Icarus (2019).99Newman Icarus (2016). 100Faulk NatGeo (2017).101Brown Nature (2008). 102Mastrogiuseppe GRL (2014). 103Mastrogiuseppe IEEE (2016) 104Mastrogiuseppe EPSL (2018). 105Mastrogiuseppe Icarus (2018).106Stofan Nature (2007). 107Stephan GRL (2010). 108Hayes JGRP (2010). 109Hayes Icarus (2011). 110Michaelides Icarus (2016). 111Birch Icarus (2017). 112Barnes PS (2014). 113Hofgartner NatGeo (2014). 114Hofgartner Icarus (2016). 115Cornet JGRP (2015). 116MacKenzie NatAstro (2019). 117Karkoschka Icarus (2016). 118Barnes JGRP (2007). 119Lorenz (2008). 120Burr GRL (2009). 121Burr Icarus (2013). 122Le Gall (2010). 123Cartwright (2011). 124Black JGRP (2012). 125Langhans Icarus (2013). 126Birch Icarus (2016). 127Radebaugh Geo. Soc. Lon. (2016). 128Cartwright Icarus (2017). ${ }_{129}$ Wasiak P\&SS (2013). 130Poggiali GRL (2016). 131Barnes Icarus (2008). 132Bonnefoy Icarus (2016). 133Soderblom P\&SS (2007). 134Le Gall Icarus (2011). 135Le Gall Icarus (2014). 136Rodriguez Icarus (2014). 137Brossier JGRP (2018). 138Radebaugh Icarus (2008). 139Radebaugh Geomorph. (2010). 140Lorenz GRL (2009). 141Malaska Icarus (2016).142Telfer JGRP (2019). 143Malaska Icarus (2016). 144Lopes Icarus (2016). 145Lopes Nat Astro (2019). 146Solomonidou JGRP (2018). 147Soderblom Icarus (2009).148Griffith Nat Astro (2019). 149Méndez Harper Nat Geo (2017).150Maynard-Casely Am Min: JEPM (2018).151Yu JGRP (2018). 152Yu EPSL (2020). 153Rodriguez NatGeo (2018). 154Corlies GRL (2017).155Aharonson NatGeo (2009). 156Hayes GRL (2017). 157Lora Icarus (2014). 158MacKenzie Icarus (2014). 159Birch Icarus (2018). 160Nimmo JGRP (2016). 161Iess Science (2012). 162Mitri Icarus (2014). 163Baland A\&A (2011). 164Baland Icarus (2014). 165Nimmo Icarus (2010). 166Hemingway Nature (2013). 167Lefevre Icarus (2014). 168Noguchi Icarus (2020). 169Castillo-Rogez GRL (2010). 170Gao Icarus (2013). 171Chen Icarus (2014). 172O'Rourke Icarus (2014). 173Leitner Icarus (2019). 174Vance JGRP (2018). 175Tobie ApJ (2012). ${ }_{176}$ Niemann Nature (2005). 177Glein Icarus (2015). 178Glein Icarus (2017). 179Marounina Icarus (2015). 180Marounina Icarus (2018). 181Miller ApJ (2019). 182Journaux Space Sci Rev (2020). 183Choblet Icarus (2017). 184Kalousová Icarus (2018). 185Journaux EPSL (2017). 186Kalousová GRL (2018). 187Journaux JGRP (2020). 188Journaux Icarus (2013). 189Hoehler Astrobio (2007). 190Shock Astrobio (2007). 191Domagal-Goldman Astrobio (2016). 192Miteva Env Microbio (2009). 193Price FEMS Microbio Eco (2007). 194Barletta Polar Record (2012). 195Cottini P\&SS (2012). 196Lopes Icarus (2007). 197Lopes JGRP (2013). 198Solomonidou JGRP (2014). 199Mitri Icarus (2008). 200Moore Icarus (2011). 201Lorenz GRL (2007). 202Le Mouélic JGRP (2008). 203Soderblom Icarus (2010). 204Neish P\&SS (2012). 205Neish Icarus (2013). 206Neish Icarus (2014). 207Neish GRL (2015). 208Neish Icarus (2016). 209Werynski Icarus (2019). 210Artemieva Icarus (2003). 211Artemieva Icarus (2005). 212O’Brien Icarus (2005). 213Neish Int J of Astrobio (2006).214Davies Icarus (2010).215Davies Icarus (2016).216Neish Astrobio (2008). 217Neish Icarus (2009). 218Neish Astrobio (2010). 219Neish Astrobio (2018). 220Orgel Ori of Life and Evol of the Biosph (1998). 221Nimmo Nature (2016). 222Neveu Nat Astro (2019). 223Benner Current Op in Chem Bio (2004). 224Lv Astrobio (2017). 225McKay Life (2016). 226Stevenson Science Advances (2015). 227Rahm PNAS (2016). 228Sandström Sci Adv (2020). 229 Cordier P\&SS (2012). 230Cordier Icarus (2016). 231Cordier Nat Astro (2017). 232Cordier AJ (2018). 233Hodyss GRL (2013). 234Corrales J Chem Phys (2017). 235Malaska Icarus (2017). 236Hartwig Icarus (2018). 237Czaplinski ACS Earth Space Chem. (2019). 238Farnsworth GRL (2019). 239Lorenz Johns Hopkins APL Tech Dig (2018). 240Turtle LPSC (2018). 241Stähler JGRP (2018). 242Smith Icarus (2016). 243West Icarus (2016). 244Le Mouélic Icarus (2018). 245Vinatier Icarus (2018). 246Lemmon Icarus (2019). 